\title{
A potential shock-reducing contingency in the backshock technique: Implications for learned helplessness
}

\author{
W. JEFFREY WILSON and LARRY L. BUTCHER \\ University of California, Los Angeles, California 90024
}

\begin{abstract}
Electric shocks were delivered to rats through a subcutaneously implanted back electrode. Experiment 1 evaluated the relationship between number of paws grounded and total power dissipated in the rat. In Experiment 2, the threshold of shock-induced vocalization, a putative index of aversiveness, was found to be positively correlated with the number of paws grounded. These findings suggest that when the backshock technique is used, the aversiveness of shock potentially can be modified by the posture adopted by the experimental animal. Caution should be exercised, therefore, in attributing deficits in escape behavior following inescapable shock administered with back electrodes to learned helplessness.
\end{abstract}

Overmier and Seligman (1967) found that exposure to inescapable shock can produce deficits in subsequent escape behavior in the dog. Similar effects have been demonstrated in the rat (Maier, Albin, \& Testa, 1973; Seligman \& Beagley, 1975), the cat (Thomas \& DeWald, 1977), and the goldfish (Padilla, Padilla, Ketterer, \& Giacolone, 1970). As a result of such studies, a learned helplessness hypothesis has been developed which holds that helplessness will ensue following sufficient exposure to an aversive stimulus that cannot be controlled by the subject. Maier and Seligman (1976) provide a comprehensive review of the learned helplessness literature.

The aversive stimulus most commonly employed in studies of learned helplessness is electric shock. Many recent studies have been carried out on the rat in which shock is delivered according to a procedure introduced in 1972 by Looney and Cohen (e.g., Beagley \& Beagley, 1978; Childress \& Thomas, 1979; Rosellini, 1978; Rosellini \& Seligman, 1978; Seligman \& Beagley, 1975; Seligman, Rosellini, \& Kozak, 1975). In this technique, a safety-pin electrode is implanted subcutaneously in the back of a rat. One lead from the shock generator is attached directly to this electrode, and the other is connected to the grid floor of the experimental chamber. The rat is thereby placed

This research was conducted in partial fulfillment of the requirements for the degree of Master of Arts in psychology (W.J.W.). It was supported by Grant NS 10928 from the U.S. Public Health Service to L.L.B., who is also affiliated with the Brain Research Institute, UCLA. The authors wish to express their appreciation to J. Perry Jaster for technical assistance in the execution of this research and to Eric W. Holman for useful criticism of an early version of this manuscript. Address all correspondence to $W$. Jeffrey Wilson, Department of Psychology, UCLA, 405 Hilgard Ave., Los Angeles, California 90024. in direct series with the shock generator. A constantcurrent shock source, one in which a high resistance is placed in series with the rat, ensures that variations in the resistance of the rat caused by its behavior have a negligible effect on the intensity of the shock.

Because learned helplessness is thought to occur as a result of repeated exposure to an uncontrollable aversive stimulus, this constant-current backshock technique is appropriate in studies of learned helplessness only if the aversiveness that it produces is unmodifiable. Such unmodifiability has never been demonstrated directly. The assumption underlying the use of this technique is that the aversiveness of electric shock is directly proportional to the intensity of the current flowing through the experimental animal. This is not necessarily the case. Campbell and Masterson (1969) provided some evidence in support of such an assumption, but they suggested further that the area of contact between the subject and the electrodes must also be considered. Early data relevant to this latter consideration are those of Forbes and Bernstein (1935) and Jackson and Riess (1934), who concluded from research done on humans that the aversiveness of electric shock is directly related to the current density, that is, the current per unit area of electrode contact, as opposed to total current intensity. Mowrer and Miller (1942) made a similar observation with regard to rats shocked through a grid floor.

In related experiments, Forbes and Bernstein (1935) noted that the power of the electric shock, equal to current times voltage, is better correlated with the subjective experiences of pain reported by their human subjects than are either the current or the current density. This suggests that the aversiveness of electric shock is directly related to the power dissipated in the experimental animal (see also Campbell 
\& Teghtsoonian, 1958; Hill, Flanary, Kornetsky, \& Wilker, 1952).

While the backshock technique ensures that the intensity of the current is constant and unmodifiable, it does not control either the current density or the power. Because experimental animals are able to modify these parameters of the shock by varying the number of paws grounded (i.e., touching the grids), they can presumably modify the aversiveness of the shock. The present studies address these issues.

\section{EXPERIMENT 1}

In the first experiment, variations in power were examined as a function of the number of paws grounded. In order to gain the necessary control over the number of paws grounded, this and the following experiment employed a method in which the subject was restrained and electrodes were attached directly to the subject's paws.

\section{Method}

Subjects. The subjects were eight female albino rats of the Sprague-Dawley strain, approximately 120 days old when the experiment was conducted. They were housed in individual cages with access to food and water ad lib. The experiment was performed during the dark phase of a reversed 12-h-light/12-h-dark cycle.

Apparatus. The shock source was a transformer, adjustable from 0 to $600 \mathrm{~V}$, which delivered $60-\mathrm{Hz}$ ac to the subject through a $510-k \Omega$ resistor. One lead from this shock source ended in an alligator clip, the other in a female banana plug.

Each of four identical paw electrodes was constructed by soldering one end of a length of No. 22 AWG wire to a polished copper penny and attaching the other end to a stackable banana plug.

A wooden support, as described in Oleson and Liebeskind (1976), was used to restrain the rat. This support held the animal in place with Velcro straps without blocking access to its feet.

Measurements of current were made with a Tektronix DM502 digital multimeter. An RCA WV547A voltmeter was used to measure voltage changes.

Procedure. Approximately 1 week before the experiment, each animal was anesthetized by ether inhalation. One prong of a safetypin electrode was inserted under the skin on a shaved portion of the rat's upper back, as described in Looney and Cohen (1972).

On the day of the experiment, each subject was anesthetized by an intraperitoneal injection of sodium pentobarbital at a dosage of $42 \mathrm{mg} / \mathrm{kg}$ of body weight. The rat was then strapped to the wooden support, and the copper-penny electrodes were attached to each foot with electrode paste and adhesive tape.

The stackable banana plugs that were attached to the paw electrodes allowed simulation of each combination of paws that an unrestrained rat might ground in a grid-floor experimental chamber. For example, stacking all four banana plugs and attaching them to the female lead from the shock source approximated the condition in which the rat has all four paws in contact with a grid floor. Similarly, attaching only the electrodes from the hindpaws to the shock source approximated the condition in which the rat is rearing. For one-half of the subjects, each paw and every combination of paws was tested in ascending order: each paw alone, every combination of two paws, each combination of three paws, and finally all four paws together. The descending order was employed for the remaining experimental animals.

The following procedure was used. The electrodes from the desired combination of paws were plugged into the lead from the shock source. Current was adjusted to $1.000 \mathrm{~mA}$ by placing the Tektronix multimeter in series with the alligator clip lead from the shock source and the back electrode and varying the output of the transformer. This level of shock was chosen because it is commonly used in studies employing the backshock technique. With the alligator clip lead attached directly to the back electrode, the voltage decrement across the whole rat was measured between the back electrode and the female banana plug lead from the shock source. The current flowing through each paw was measured by putting the Tektronix multimeter in series with the electrode from that paw and the female banana plug. This sequence was repeated for each paw or combination of paws, in the order described above. The interval between measurements was approximately $1 \mathrm{~min}$.

Because there is only one way in which all four paws can be grounded simultaneously, the value of current density recorded for each subject in this condition consisted of the mean of the measurements taken for each paw, that is, the mean of four measurements. There are four ways in which three paws can be grounded, so the value recorded for each subject in this condition was the mean of 12 measurements. Similarly, six ways of grounding two paws yielded 12 measurements in this condition. Because the voltage decrease was measured across the whole rat rather than across each paw, the values recorded for each subject for the 4-, 3-, 2-, and 1-paw conditions were the means of, respectively, $1,4,6$, and 4 measurements.

The total power $(\mathrm{P})$ dissipated in the rat was computed by the formula $\mathrm{P}=\mathrm{I}_{\mathrm{T}} \times \mathrm{V}$, where $\mathrm{I}_{\mathrm{T}}$ is the total current $(1.000 \mathrm{~mA}$, in this case) and $V$ is the measured voltage decrease. The resistance (R) of the rat was determined by the formula $R=V / I_{T}$. Although in most circuits employing alternating current, impedance, which involves resistance, inductance, and capacitance, must be considered instead of resistance alone, Hill et al. (1952) demonstrated that inductance and capacitance are negligible in biological circuits when $60-\mathrm{Hz}$ ac is used. In the present situation, therefore, impedance equals resistance and no consideration need be given to the inductance or capacitance of the rat.

\section{Results and Discussion}

Because total current intensity was held constant at $1.000 \mathrm{~mA}$, the average current flowing through each paw of the animal must be proportional to the number of paws grounded, as, indeed, was the case. Power, voltage, and resistance all changed as the number of paws that were grounded changed, although total current was held constant. The power data (in milliwatts) appear in Table 1; voltage and resistance, which are linearly related to power, are not reported. The data were evaluated using an analysis of variance with order of testing the paws (ascending vs. descending) as the between-subjects factor and number of paws grounded as the withinsubject factor.

There was a significant relationship between number of paws grounded and power dissipated in the rat $[F(3,18)=435.36, p<.001]$. The order of taking the measurements had a significant effect $[F(1,6)=$ $18.93, \mathrm{p}<.01]$ and interacted significantly with the number of paws grounded $[\mathrm{F}(3,18)=7.79, \mathrm{p}<.01]$. In light of this interaction, multiple comparisons of the means for number of paws grounded were made separately for each order using the NewmanKeuls procedure (Keppel, 1973). For both the ascending and the descending orders, the consecutive means 
Table 1

Means and Standard Deviations of Power (in Milliwatts) for Each Number of Paws Grounded for Ascending and Descending Orders

\begin{tabular}{|c|c|c|c|c|c|c|c|c|}
\hline \multirow[b]{3}{*}{ Order } & \multicolumn{8}{|c|}{ Number of Paws Grounded } \\
\hline & \multicolumn{2}{|c|}{ One } & \multicolumn{2}{|c|}{ Two } & \multicolumn{2}{|c|}{ Three } & \multicolumn{2}{|c|}{ Four } \\
\hline & Mean & SD & Mean & SD & Mean & SD & Mean & SD \\
\hline Ascending & 9.72 & .916 & 5.84 & .448 & 4.31 & .324 & 3.45 & .339 \\
\hline Descending & 10.08 & .259 & 7.25 & .603 & 6.04 & .514 & 5.23 & .427 \\
\hline
\end{tabular}

differed significantly. In both cases, there was a decrease in the total power dissipated as the number of paws increased. The results for the ascending order were: one vs. two paws, $\mathrm{F}(1,3)=201.93, \mathrm{p}<.01$; two vs. three paws, $F(1,3)=158.92, p<.01$; three vs. four paws, $F(1,3)=4331.33, p<.01$. Those for the descending order were: one vs, two paws, $F(1,3)$ $=224.32, p<.01$; two vs. three paws, $F(1,3)=107.74$, $\mathrm{p}<.01$; three vs. four paws, $\mathrm{F}(1,3)=23.43, \mathrm{p}<.05$.

Comparisons of the means for order at each level of the number of paws grounded revealed that the mean for the descending order was significantly greater than that for the ascending order for the four-, three-, and two-paw conditions $[\mathrm{Fs}(1,6)=42.36$, 32.88 , and 14.01 , ps $<.01, .01, .05$, respectively], but not for the one-paw condition $[F(1,6)=.60$, $p>.05]$.

This experiment demonstrates that when the total current flowing through the rat is held constant, power is inversely related to the number of paws that are grounded. This result follows from the fact that, as the number of paws grounded decreases, the resistance presented by the animal increases. This increase in resistance raises the voltage required to maintain current at $1.000 \mathrm{~mA}$, consequently raising the power dissipated in the rat. When the backshock technique is used, the rat acts much as if there were a set of resistors wired in parallel from the back electrode to the four paw electrodes. While the total current remains constant, the current density and power both decrease as the number of parallel paths increases.

\section{EXPERIMENT 2}

If the aversiveness of electric shock is related to its current density or power, then the results of the first experiment suggest that aversiveness should decrease as the number of paws grounded increases. The present experiment examined the relationship between number of paws grounded and shock-induced vocalization. Vocalization has been described as a reliable indicator that a rat is experiencing an aversive stimulus (Charpentier, 1968; Lim \& Guzman, 1968). Thus, although the aversiveness of a stimulus cannot be measured directly, it can be inferred from the extent to which the stimulus causes a rat to vocalize.

\section{Method}

Subjects. The subjects were seven female albino rats of the Sprague-Dawley strain, approximately 150 days old at the time of the experiment. They were maintained as in Experiment 1 .

Apparatus. The apparatus was the same as that used in Experiment 1 except that a switch was employed that allowed a 5.1-k $\Omega$ resistor to be substituted for the rat in the circuit. This resistor approximated the mean resistance of the rat when three paws were grounded, as determined in Experiment 1, and allowed the level of current to be adjusted without passing the current through the rat.

Procedure. Each subject was anesthetized by ether inhalation, and a safety-pin electrode was implanted as described in Experiment 1 . On the following 2 days, each subject was habituated to the restraint procedure by being strapped to the wooden support for approximately $20 \mathrm{~min}$. On the $3 \mathrm{rd}$ day, in addition to being restrained for $20 \mathrm{~min}$, the subject had the copper penny electrodes attached to its paws with electrode paste and adhesive tape. The rat's paws and legs were taped against the sides of the support to prevent the animal from removing the electrodes. By the 3 rd day, the subjects accepted this restraint without struggling

The experiment was carried out on the 4th day. Each subject was restrained, and electrodes were attached as described previously. The Tektronix multimeter was placed in series with the $510-\mathrm{k} \Omega$ resistor from the shock source and the common pole of the switch. The normally open contact of the switch was attached to the back electrode of the subject, and the normally closed contact was wired, through the $5.1-\mathrm{k} \Omega$ resistor, to the other pole of the shock source. Also attached to this pole of the shock source was a lead ending in a female banana plug, through which the paw electrode could be grounded. Thus, with the switch in its normal position, current flowed through the multimeter and the 5.1-k $\Omega$ resistor, and when the switch was in its other position, current flowed through the multimeter and the subject.

Unlike the previous experiment, which examined every possible combination of paws, the present experiment dealt only with those combinations of paws that would be grounded as a result of the rat's normal postures. Thus, each subject was tested with four paws grounded, two hindpaws and one forepaw grounded, and the two hindpaws alone grounded. Each of these conditions was tested twice for each subject, once in ascending order (2-3-4 paws) and again in descending order (4-3-2 paws). The ascending and descending orders were counterbalanced, such that three subjects received the ascending order first and four subjects received the descending order first.

The levels of current employed, in milliamps, were $0, .125$, $.250, .375, .500, .625, .750, .875,1.000,1.125,1.250$, and 1.335 , which was the highest level reliably achievable with the apparatus used.

For each combination of paws, the shocks were presented in increasing order of intensity according to the following procedure. The desired combination of paws was grounded by plugging the electrodes from those paws into the lead from the shock source. With the transformer output diverted through the $5.1-\mathrm{k} \Omega$ resistor the current was adjusted to the desired level. Approximately $30 \mathrm{sec}$ after the previous shock, the switch was thrown and the current was allowed to pass through the rat for $3 \mathrm{sec}$, timed by a slopwatch. A record was made of whether or not the rat vocalized 
Table 2

Means and Standard Deviations of Vocalization Threshold (in Milliamperes) for Each Number of Paws Grounded for Ascending and Descending Orders Under Each Sequence of Measurement and Grand Means

\begin{tabular}{|c|c|c|c|c|c|c|c|c|c|}
\hline & \multirow[b]{3}{*}{$\mathrm{n}$} & \multicolumn{6}{|c|}{ Number of Paws Grounded } & & \\
\hline & & \multicolumn{2}{|c|}{ Two } & \multicolumn{2}{|c|}{ Three } & \multicolumn{2}{|c|}{ Four } & \multicolumn{2}{|c|}{ Grand Mean } \\
\hline & & Mean & SD & Mean & SD & Mean & SD & Mean & SD \\
\hline & \multicolumn{9}{|c|}{ Ascending Order } \\
\hline First & 3 & .417 & .144 & .417 & .260 & .792 & .577 & .542 & .375 \\
\hline \multirow[t]{2}{*}{ Second } & 4 & .709 & .465 & .740 & .461 & .949 & .449 & .799 & .429 \\
\hline & \multicolumn{9}{|c|}{ Descending Order } \\
\hline First & 4 & .531 & .295 & .855 & .591 & .918 & .482 & .768 & .462 \\
\hline \multirow[t]{3}{*}{ Second } & 3 & .500 & .217 & .875 & .433 & .917 & .382 & .764 & .367 \\
\hline & \multicolumn{9}{|c|}{ Grand Mean } \\
\hline & & .551 & .272 & .733 & .418 & .899 & .429 & & \\
\hline
\end{tabular}

during the shock. A vocalization was defined as any audible squeal, regardless of its intensity or duration. This procedure was repeated for each level of shock. When all shock levels had been tested for one combination of paws, the next combination was tested. This was continued until every combination of paws had heen rested in both the ascending (2-3-4 paws) and descending (4-3-2 paws) orders

\section{Results and Discussion}

On each trial, the current flowing through the rat was observed. Because it rarely varied by more than $.01 \mathrm{~mA}$ from the preset level, the $5.1-\mathrm{k} \Omega$ resistor closely approximating the resistance of the rat as determined in Experiment 1, the preadjusted current levels were used in further considerations.

For each combination of paws grounded, the level of shock at and beyond which the subject vocalized to every level of shock was recorded as the vocalization threshold. If a subject failed to vocalize at any shock intensity for a given combination of paws, a threshold of $1.335 \mathrm{~mA}$ was recorded for that combination. The mean threshold for each combination of paws grounded appears in Table 2 .

An analysis of variance was performed on these data with the sequence of presenting the ascending and descending series (first or second) as the betweensubjects factor and order of testing the paws (ascending vs. descending) and number of paws grounded as the within-subject factor.

The number of paws grounded was a significant factor $[F(2,10)=5.05, p<.05]$ and did not interact with any other factor. As indicated by the grand means shown in Table 2, the vocalization threshold increased as the number of paws grounded increased. Comparisons of these grand means revealed that the threshold for the four-paw condition was significantly higher than that of the two-paw condition $[F(1,6)=$ $8.90, p<.025]$ and that of the three-paw condition $[F(1,6)=8.62, p<.05]$. The three- and two-paw conditions did not differ significantly $[F(1,6)=2.35$, $\mathrm{p}>$.05].
The only other significant effects were order $[F(1,5)$ $=13.42, \mathrm{p}<.025]$ and the interaction between order and sequence $[F(1,5)=23.66, p<.005]$. Because of the interaction, the simple effects of the two factors, represented by the grand means shown in the right column of Table 2, were evaluated. When the sequence of orders was ascending first and descending second, the vocalization threshold was lower for the ascending order $[F(1,5)=36.89, p<.01]$. However, when the descending order was presented first and the ascending order second, the vocalization thresholds did not differ $[F(1,5)=.72, p>.05]$. When the ascending order was presented first, the vocalization threshold was lower than when it was presented second $[F(1,5)=48.82, p<.01]$, but the thresholds obtained with the descending order did not depend on whether this order was presented first or second $[F(1,5)=.01, p>.05]$. These findings indicate a greater sensitivity to shock throughout the ascending order only when this order was presented first.

It seems clear from the results of this experiment that, when the backshock technique is used, there is an inverse relationship between the number of paws that are grounded and the aversiveness of electric shock, as indexed by vocalization.

\section{GENERAL DISCUSSION}

In the present experiments, the rat was restrained, and electrodes were attached directly to its paws. In this procedure, the rat's role in the electric circuit differs from that of an unrestrained rat in at least two ways. First, the amount of tissue of each paw in contact with the electrode certainly does not vary as much in the present procedure as in the case of the unrestrained rat. This was a necessary component of our procedure because we were varying systematically current density, and any variation in the surface area of each paw contacting the electrode would have altered the current density. Thus, we varied current 
density by only one means: varying the number of paws that were grounded. The unrestrained rat can do so in yet another way, varying the area of contact of each paw with the electrode. It is our contention that the two strategies produce similar variations in the aversiveness of the shock and that our restraint procedure can be generalized to the unrestrained case with validity.

The second difference between our procedure and the case of the unrestrained rat is that the making and breaking of contact between the paws and the electrodes that occurs in the freely moving rat is eliminated by restraint. Muenzinger and Walz (1932) reported that, for human subjects, the making and breaking of contact was more aversive than the maintenance of contact. If it is assumed that the same holds true for the rat, then one effect of our procedure was to eliminate one of the aversive aspects of the shock. If this is, indeed, the case, one might expect the rat in the unrestrained case to do the same, that is, to remain motionless. Although the present procedure might not generalize fully to an unrestrained rat that is making or breaking contacts, we believe that it generalizes well to the unrestrained rat in those periods of time when a contact is not being made or broken.

The present experiments further indicate that the aversiveness experienced by the rat when the backshock technique is used is predicted better by the current density or by the power of the shock than by current intensity. The results also suggest that when the backshock technique is used a contingency probably exists between the behavior of the rat and the aversiveness of the electric shock, in that the aversiveness of the electric shock can be minimized when the rat's area of contact with the grids is maximized.

With this contingency between the subject's behavior and the aversiveness of the shock, the "helplessness" that results in studies employing the backshock technique can be readily explained. Rats that are made "helpless" by this technique are usually described as remaining motionless and passive during the shock. In the absence of a contingency between the rat's behavior and the aversiveness that it experiences, the helplessness hypothesis explains these results well. Given that such a contingency exists, however, a more parsimonious explanation is that the rat is capable of learning the contingency between remaining in contact with the grids and reducing the shock's aversiveness. It would be very difficult in such a situation to distinguish between the symptoms of helplessness and the performance of this competing instrumental response.

Maier and Seligman (1976) have argued on the basis of a study by Maier (1970) that the learning of such a contingency cannot account for the helplessness phenomenon. In that study, dogs that were explicitly trained to escape shock by remaining motionless were later able to learn a novel escape task with little difficulty. That study differs from those employing the backshock technique, however, in an important aspect. For Maier's dogs, freezing was no longer an effective escape response in the test session; that is, the contingency between remaining motionless and the escape from shock was no longer in effect. The response of freezing should have extinguished, allowing the acquisition of the effective shuttling response, which is, indeed, what happened. When the backshock technique is used, remaining motionless is as effective in the test session as during training; that is, the contingency remains in effect, and there is no reason to expect the response of remaining motionless to undergo extinction. Its persistence would prevent the acquisition of the novel escape response.

The possibility that learned inactivity, freezing, or the punishment of movement is the cause of the helplessness phenomenon has been proposed by others (Anisman, deCatanzaro, \& Remington, 1978; Anisman \& Waller, 1973; Bracewell \& Black, 1974; Levis, 1976). The present explanation differs from those of other investigators in an important way, however. To the extent that any of the other explanations rely on contingencies, those contingencies are implicit in that they are thought to occur due to the fortuitous pairing of inactivity, or some other competing response, and shock offset (Anisman et al., 1978; Levis, 1976). The experiments reported herein demonstrate the potential existence of an explicit contingency between the subject's behavior and a reduction in the aversiveness experienced by the subject.

\section{REFERENCES}

Anisman, H., deCatanzaro, D., \& Remington, G. Escape performance following exposure to inescapable shock: Deficits in motor response maintenance. Journal of Experimental Psychol. ogy: Animal Behavior Processes, 1978, 4, 198-218.

Anisman, H., \& Waller, T. G. Effects of inescapable shock on subsequent avoidance performance: Role of response repertoire changes. Behavioral Biology, 1973, 9, 331-355.

Beagley, G. H., \& Beagley, W. K. Alleviation of learned helplessness following septal lesions in rats. Physiological Psychology, 1978, 6, 241-244.

Bracewfll, R. J., \& Black, A. H. The effects of restraint and noncontingent preshock on subsequent escape learning in the rat. Learning and Motivation, 1974, 5, 53-69.

Campbell, B. A., \& Masterason, F. A. Psychophysics of punishment. In B. A. Campbell \& R. M. Church (Eds.), Punishment and aversive behavior. New York: Appleton-Century-Crofts, 1969.

Campbeli, B. A., \& Teghtsoonian, R. Electrical and behavioral effects of different types of shock stimuli on the rat. Journal of Comparative and Physiological Psychology, 1958, 51, 185-192.

Charpentrit, J. Analysis and measurement of pain in animals: A new conception of pain. In A. Soulairac, J. Cahn, \& J. Charpentier (Eds.), Pain. New York: Academic Press, 1968. 
Childress, A. R., \& Thomas, E. A comparison of central averive stimulation and peripheral s.locks in the production of learned helplessness. Physiological Psychology, 1979. 7, 131-134.

Forbes, T. W., \& Bernstein, A. L. The standardization of sixty-cycle electric shock for practical use in psychological experimentation. Journal of General Psychology, 1935, 12, 436-442.

Hili, H. E., Flanary, H. G., Kornetsky, C. H., \& Wilker. A. Relationship of electrically induced pain to the amperage and wattage of shock stimuli. Journal of Clinical Investigation, $1952,31,464-472$.

J ACKSON, T. A., \& RiESS, B. F. Electric shock with different size electrodes. Journal of Genetic Psychology, 1934, 45, 262-266.

KEPPEL, G. Design and analysis: A researcher's handbook. Englewood Cliffs, N.J: Prentice-Hall, 1973.

LEvis, D. J. Learned helplessness: A reply and an alternative S-R interpretation. Journal of Experimental Psychology: General, 1976, 105, 47-65.

Lim. R. K. S., \& Guzman, F. Manifestations of pain in analgesic evaluation in animals and man. In A. Soulairac, J. Cahn, \& J. Charpentier (Eds.), Pain. New York: Academic Press, 1968.

Looney, T. A., \& Cohen, P. S. Retardation of jump-up escape responding in rats pretreated with different frequencies of noncontingent electric shock. Journal of Comparative and Physiological Psychology, 1972, 78, 317-322.

MAlkr. S. F. Failure to escape traumatic shock: Incompatible skeletal motor response or learned helplessness? Learning and Motivation, 1970, 1, 157-170.

Maier, S. F., Albin, R. W., \& Testa, T. J. Failure to learn to escape in rats previously exposed to inescapable shock depends on nature of escape response. Journal of Comparative and Physiological Psychology, 1973, 85, 581-592.

Maier. S. F., \& Seligman, M. E. P. Learned helplessness: Theory and evidence. Journal of Experimental Psychology: General, 1976, 105, 3-46.
Mowrer, O. H., \& Miller, N. E. A multi-purpose learning demonstration apparatus. Journal of Experimental Psychology, 1942, 31, 163-170.

Muenzinger, K. F., \& Walz, F. C. An analysis of the electrical stimulus producing a shock. Journal of Comparative Psychology. $1932,13,157-171$.

Oleson, T. D., \& Liebeskind, J. C. Modification of midbrain and thalamic evoked responses by analgesic brain stimulation in the rat. In J. J. Bonica \& D. Albe-Fessard (Eds.), Advances in pain research and therapy (Vol. 1). New York: Raven Press, 1976.

Overmier, J. B., \& Seligman, M. E. P. Effects of inescapable shock upon subsequent escape and avoidance behavior. Journal of Comparative and Physiological Psychology, 1967, 63, 28-33.

Padill.a, A. M., Padilla, C., Ketterer, T., \& Giacolone, D. Inescapable shocks and subsequent avoidance conditioning in goldfish (Carrasius auratus). Psychonomic Science, 1970, 20, 295-296.

Rosellini, R. A. Inescapable shock interferes with the acquisition of an appetitive operant. Animal Learning \& Behavior, $1978,6,155-159$.

Rosellini, R. A., \& Seligman, M. E. P. Role of shock intensity in the learned helplessness paradigm. Animal Learning \& Behavior, 1978, 6, 143-146.

Seligman, M. E. P., \& Beagley, G. Learned helplessness in the rat. Journal of Comparative and Physiological Psychology, 1975, 88, 534-541.

Seligman, M. E. P., Rosellini, R. A., \& Kozak, M. Learned helplessness in the rat: Reversibility, time course, and immunization. Journal of Comparative and Physiological Psychology, $1975,88,542-547$.

Thomas, E., \& DeWald, L. Experimental neurosis. In J. D. Maser \& M. E. P. Seligman (Eds.), Psychopathology: Experimental models. San Francisco: Freeman, 1977.

(Received for publication December 7, 1978; revision accepted March 5, 1980.) 